\title{
Aqueous Behaviour of Chitosan
}

\author{
D. P. Chattopadhyay ${ }^{1}$ and Milind S. Inamdar ${ }^{2}$ \\ ${ }^{1}$ Department of Textile Chemistry, Faculty of Technology and Engineering, The Maharaja Sayajirao University of Baroda, \\ Vadodara 390001, India \\ ${ }^{2}$ Faculty of Textile Processing, Sarvajanik College of Engineering and Technology, Surat 395001, India
}

Correspondence should be addressed to D. P. Chattopadhyay, dpchat6@gmail.com

Received 27 September 2010; Revised 6 December 2010; Accepted 30 December 2010

Academic Editor: Peter J. Halley

Copyright (C 2010 D. P. Chattopadhyay and M. S. Inamdar. This is an open access article distributed under the Creative Commons Attribution License, which permits unrestricted use, distribution, and reproduction in any medium, provided the original work is properly cited.

\begin{abstract}
Chitosan, a versatile biopolymer, finds numerous applications in textile processing unit operations such as preparation, dyeing, printing, and finishing. However, the accessibility of this biopolymer by the textile material depends on the viscosity of its solution which in turn is a function of its molecular weight. In this work, therefore, the effect of molecular weight, storage life, presence of electrolyte, and particle size of chitosan on its viscosity was investigated. Chitosan of different molecular weights was synthesized by nitrous acid hydrolysis of parent chitosan solution. The synthesized low molecular weight products were analysed by FTIR spectroscopy. Chitosan of nanoconfiguration was prepared by Ionotropic gelation method and characterized by particle size analyzer. The viscosity of different chitosan solutions was determined using Ubbelohde capillary viscometer. As an extension to this study, the chelation property of chitosan was also evaluated.
\end{abstract}

\section{Introduction}

Chitosan, a versatile biopolymer derivative, is obtained by alkaline deacetylation of chitin. The distribution of the precursor, chitin, in nature is ubiquitous among the shells of crustaceans such as crabs, shrimps, and lobsters as well as in the exoskeleton of marine zoo-plankton including coral, jellyfish, and squid pens. Chemically, chitosan is a linear (1-4) linked 2 -amino-2-deoxy- $\beta$-d-glucan (i.e., $\beta$-dglucosamine) in the chair ${ }^{4} \mathrm{C}_{1}$ conformation, Figure 1 . The structure of chitosan closely resembles that of cellulose, except an hydroxyl group at C2 position in cellulose being replaced by amino group in chitosan. Indeed, it is a copolymer of glucosamine and $\mathrm{N}$-acetyl glucosamine units. Chitosan exhibits several valuable inherent properties such as antibacterial, antifungal, antiviral, antacid, nontoxic, total biodegradable as well as film formation, fibre formation, and hydrogel formation properties [1,2]. By virtue of these properties, chitosan has prospective applications in many fields such as medical, waste water treatment, cosmetics, dentifrices, food, agriculture, pulp and paper, and textile industries $[3,4]$.
In textiles, it finds applications in the primary production of fibres (useful for sutures, wound dressings, etc.), in the manufacture of textile auxiliary chemicals and finishing agents [5-7]. Investigations have shown that it can improve the dye uptake of cotton fibre [8]. It can also be used as a dye-fixing agent, for shade and naps coverage, to improve the fastness of dyed fabrics, as a binder in pigment printing, as a thickener in printing [9]. It is also found that the treatment of wool with chitosan minimizes its felting problem. The bioadhesive and cationic nature of chitosan enables it to form a strongly adhered film on individual fibres and prevent their entanglements [10]. By virtue of its antibacterial property, chitosan can prevent garments to develop bad odour [1113].

Chitosan when dissolved in acidic solution gives viscous solutions. The viscosity determines the extent of penetration of chitosan into the fabric structure. In solutions, chitosan in suitable conditions shows hydrogel formation and viscoelastic behaviour [14-17]. Its rheological behaviour to characterize its usefulness as thickener in printing paste is reported [18]. However its aqueous behaviour pertaining to various unit operations of textile processing is hardly 


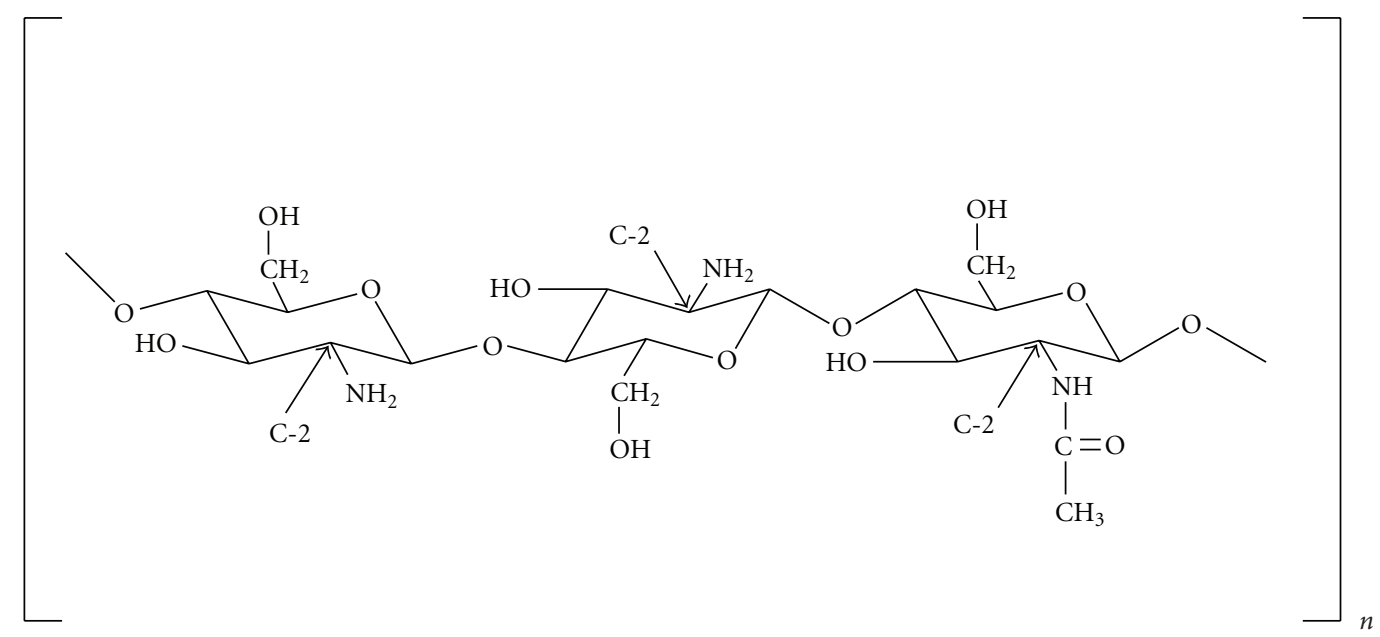

FIgURE 1: Structure of chitosan.

TABle 1: Specifications of different chitosans.

\begin{tabular}{lcc}
\hline Particulars & \multicolumn{2}{c}{ Specifications } \\
\hline Nomenclature & CHT1 & CHT3 \\
DAC Value (\%) & 89.03 & 90 \\
Viscosity (m Pa·s ) & 183 & 22 \\
Molecular Weight & 654,127 & 135,839 \\
\hline
\end{tabular}

reported in the literature. The present research work, therefore, mainly aimed at investigating the aqueous behaviour of chitosan with respect to its molecular weight, concentration and particle size on viscosity and stability of its solution. The effect of electrolytes such as sodium acetate is also reported. The work is also extended to study the chelation behaviour of chitosan.

\section{Materials and Methods}

2.1. Materials. Chitosan samples of different molecular weights were purchased from M/s Marine chemicals, Kerala state, India (CHT1) and M/s Mahtani Chitosan Pvt. Ltd., Gujarat State, India (CHT3). The molecular weights of these samples were determined viscometrically. The specifications of different chitosans are given in Table 1 .

Other chemicals such as acetic acid, sodium acetate, sodium hydroxide, trisodium polyphosphate (TPP), methanol, and tetra sodium salt of ethylene diamine tetra acetic acid ( $\left.\mathrm{Na}_{4} \mathrm{EDTA}\right)$ used were of analytical grade of reputed companies.

2.2. Synthesis of Low Molecular Weight Chitosan. Low molecular weight chitosans were synthesized by controlled hydrolysis of chitosan using nitrous acid as depolymerising agent. A $2 \%$ solution of chitosan in acetic acid was prepared. Predissolved dilute solution of sodium nitrite was then added gradually to chitosan solution and stirred for two hrs at $30^{\circ} \mathrm{C}$ to get desired viscosity level. The depolymerized chitosan was then precipitated out by caustic solution and washed to neutral $\mathrm{pH}$. The precipitates of chitosan was then washed 3 to 4 times with methanol and dried at $60^{\circ} \mathrm{C}$. The product obtained from CHT1 is termed as $\mathrm{CHT} 2$ whereas the products obtained from CHT3 are termed as CHT4 \& CHT5.

2.3. Determination of Molecular Weight and Viscosity. The molecular weight and viscosity behaviour of chitosan was determined using Ubbelohde capillary viscometer (No 1A) at $30^{\circ} \mathrm{C}$ having flow time for distilled water, $T_{0}=15.57$ seconds. Chitosan solutions of different concentrations in $0.25 \mathrm{M}$ acetic acid and $0.25 \mathrm{M}$ sodium acetate were prepared. During preparation, all the solutions were magnetically stirred for 1 hour to ensure proper dissolution of chitosan and were filtered using Whatman filter paper no 4 . The flow times of chitosan solutions and solvent were recorded in triplicate and the average value was calculated. The intrinsic viscosity $[\eta]$ was calculated graphically by extrapolating the curve of reduced viscosity versus concentration to zero concentration. The molecular weight was then calculated by using Mark-Houwink equation (1) [19].

2.4. FTIR Analysis. FTIR spectra of chitosan and depolymerised chitosan samples were recorded on a Thermo Nicolet iS10 Smart ITR spectrophotometer (Thermo Fisher Scientific, USA), equipped with an OMNIC-Software, a DTGS detector, and a Ge-on-KBr beamsplitter (4000$\left.500 \mathrm{~cm}^{-1}\right)$.

2.5. Preparation of Nanochitosan and Its Characterization. Chitosan (CHT3) was dissolved in acetic acid solution and optimized quantity of TPP was added dropwise with rapid stirring (about $400 \mathrm{rpm}$ ) to obtain an opalescent solution containing chitosan $25 \mathrm{mg} / \mathrm{dL}$ and TPP $3.75 \mathrm{mg} / \mathrm{dL}$. The sample was allowed to stand overnight and filtered through sintered glass filter of porosity grade G3 and preserved in refrigerator. The prepared chitosan was coded as CHT3N.

The particle size and size distribution of the chitosan were analyzed on the particle size analyser (Model: Zetasizer Nano-ZS90, Make: Malvern Instruments Ltd, UK). 
2.6. Evaluation of Metal Ion Chelation Value in Water. The amount of $\mathrm{Ca}^{+2}$ as calcium chloride in water for chelation study were determined by volumetric analysis of $50 \mathrm{~mL}$ sample against $0.02 \mathrm{M} \mathrm{Na}_{2}$ EDTA solution using Eriochrome black $\mathrm{T}$ indicator.

\section{Results and Discussion}

3.1. Effect of Molecular Weight and Concentration. Chitosan is characterized mainly by two variables, namely, degree of deacetylation (DAC value) and the molecular weight. Degree of deacetylation determines the number of free amino groups present in the chitosan macromolecule, which in turn determines the functionality, polarity, and water solubility of the polymer. On the other hand, molecular weight determines the strength of its fiber/film and viscosity of its solution. Different molecular weight chitosan can be obtained by controlled depolymerization by methods such as acid hydrolysis $\left(\mathrm{HCl}, \mathrm{HNO}_{2}\right.$, etc.), free radicals $\left(\mathrm{H}_{2} \mathrm{O}_{2}\right.$, $\mathrm{K}_{2} \mathrm{~S}_{2} \mathrm{O}_{8}$ ), enzymatic, radiations (UV, $\gamma$ rays) ultrasound, microwave, and thermal treatments [3, 20-24].

When chitosan solution is treated with nitrous acid, produced from acidic solution of sodium nitrite, it undergoes deamination reaction with subsequent cleavage of $\beta$ glycosidic linkages. The reaction scheme and FTIR spectra are illustrated in Figures 2 and 3, respectively.

It envisaged from Figure 3 that the IR spectra of chitosan and depolymerised chitosan are almost similar which indicates that the process of depolymerisation caused no chemical change in the structure of the polymer except reduction in molecular weight which is evident from the change in viscosity.

The viscosity of polymer solution, at the molecular level, is a direct measure of the hydrodynamic volume of the polymer molecules which in turn is governed by the molecular size or the chain length and hence the molecular weight [25]. Therefore, viscosity, measured by capillary viscometer, is widely employed to determine the average molecular weight of a polymer by using the famous MarkHouwink equation (1)

$$
[\eta]=k\left[M_{V}\right]^{\alpha},
$$

where $M_{V}$ is the viscosity average molecular weight of polymer, $\alpha$ and $k$ are constants $\left(\alpha=0.83\right.$ and $k=1.4 \times 10^{-4}$ for $0.25 \mathrm{M}$ acetic acid and $0.25 \mathrm{M}$ sodium acetate solvent system) $[26]$, and $[\eta]$ is the limiting viscosity number or intrinsic viscosity and can be determined from [19]

$$
[\eta]=\lim _{C \rightarrow 0} \frac{\left(\eta-\eta_{s}\right)}{\eta_{s} C}
$$

where $\eta$ is the solution viscosity and $\eta_{s}$ is the solvent viscosity and $C$ is the solution concentration. As indicated in (2), when $\left(\eta-\eta_{s}\right) / \eta_{s} C$, that is, reduced viscosity $\left(\eta_{\text {red }}\right)$ is plotted against concentration $(C)$, the intercept corresponds to intrinsic viscosity $[\eta]$. Such plots for different grades of chitosan are
TABLE 2: Intrinsic viscosity and viscosity average molecular weight of different grades of chitosan.

\begin{tabular}{lcc}
\hline Chitosan & Intrinsic viscosity $[\eta], \mathrm{dL} / \mathrm{g}$ & Molecular weight, $M_{V}$ \\
\hline CHT-1 & 9.40 & 654,127 \\
CHT-2 & 4.72 & 285,231 \\
CHT-3 & 2.55 & 135,839 \\
CHT-4 & 1.50 & 71,676 \\
CHT-5 & 0.535 & 20,698 \\
\hline
\end{tabular}

shown in Figure 4 and the corresponding intrinsic viscosities and calculated molecular weights are presented in Table 2.

It can be seen from Figure 4 that the curves of high molecular weight chitosan, namely, CHT-1, 2, and 3, do not strictly follow the linearity of (2). They show the inflection at a certain critical concentration $\left(C^{*}\right)$ and then after the curves bend upwards. Further, the value of $C^{*}$ increases with decrease in molecular weight and ultimately the curves flatten for lower molecular weight chitosans. This can be explained as follows: when chitosan or any other polymer is added into a solvent, the solvent gradually diffuse into the polymer aggregates resulting into the swelling of the polymer. As swelling continues, the segments of the polymer are solvated and loosened out. Since the molecules in a solid polymer remains entangled with neighbouring ones, polymer molecules during dissolution diffuse out as bunches of entangled molecules. Even when all chain segments of a polymer molecule in solution are unfolded and fully solvated, the molecules do not assume the shape of an extended straight chain but present in a coil form. These coils or aggregates offer resistance for the mobility or flow of molecules and hence impart viscosity $[19,27]$. When the molecular size and concentration is increased, as in our case, the extent of entanglement is increased. In other word, the critical concentration $\left(C^{*}\right)$ is, indeed, the "overlap" concentration. When $C>C^{*}$ the intermolecular entanglements or aggregation, predominate and preclude the overall molecular motion of the polymer, while individual polymer molecules are statistically separated from other molecules at $C<C^{*}$. Thus, the critical concentration $\left(C^{*}\right)$ is a measure of molecular size and conformation of a polymer. The higher the molecular weight and the more rigid is the conformation, the lower the value of $C^{*}$. The results are in close agreement with the earlier reports $[15,28]$.

3.2. Effect of Storage Time on Viscosity of Chitosan Solution. Polymeric chemicals are generally applied to textiles by padding technique where it is required to prepare a standing bath. Thus the chemical remains in contact with water for a longer period. As biodegradability of chitosan is a well-known phenomenon the effect of storage time on the stability of its solution was studied in terms of change in solution viscosity. It is seen from Figure 5 that the viscosity of chitosan solution is adversely affected with increase in storage time.

It seems that the initial molecular weight and the concentration of polymer has the influence on the stability 
$\mathrm{NaNO}_{2}+\mathrm{H}^{+} \longrightarrow \mathrm{HNO}_{2}+\mathrm{Na}^{+}$

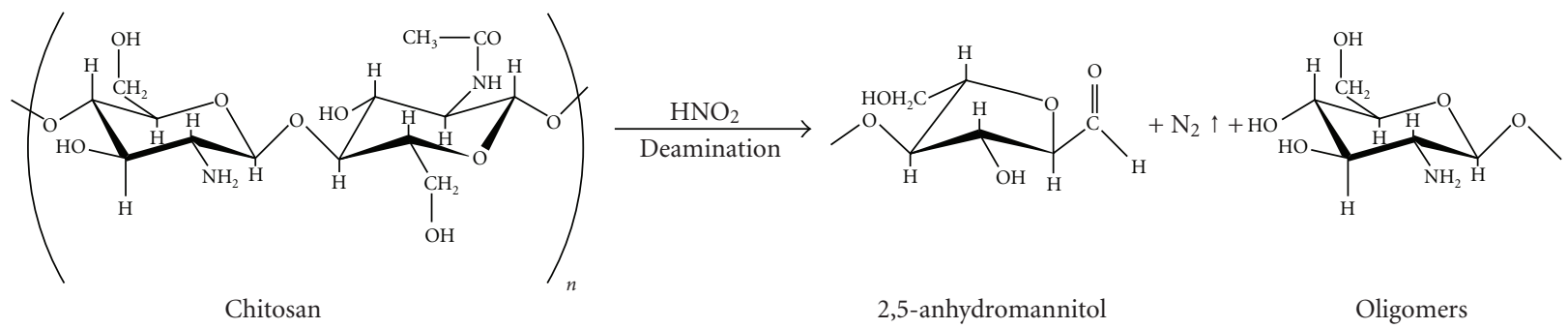

FIGURe 2: Depolymerization of chitosan by nitrous acid.

TABLE 3: Chelation property of chitosan.

\begin{tabular}{lcccccc}
\hline Treatment & Concentration, gpl & \multicolumn{4}{c}{ Amount of $\mathrm{Ca}^{+2}$ ions (ppm) chelated after: } \\
& & $2 \mathrm{hrs}$ & $24 \mathrm{hrs}$ & 48 hrs & 72 hrs & $96 \mathrm{hrs}$ \\
\hline $\mathrm{Na}_{4}$ EDTA & 1 & 405 & 426 & 435 & 450 & 452 \\
$\mathrm{CHT1}$ & 1 & 177 & 208 & 232 & 269 & 293 \\
$\mathrm{CHT3}$ & 1 & 182 & 187 & 185 & 189 & 196 \\
\hline
\end{tabular}

Initial concentration of $\mathrm{Ca}^{+2}$ in water $=625 \mathrm{ppm}$.

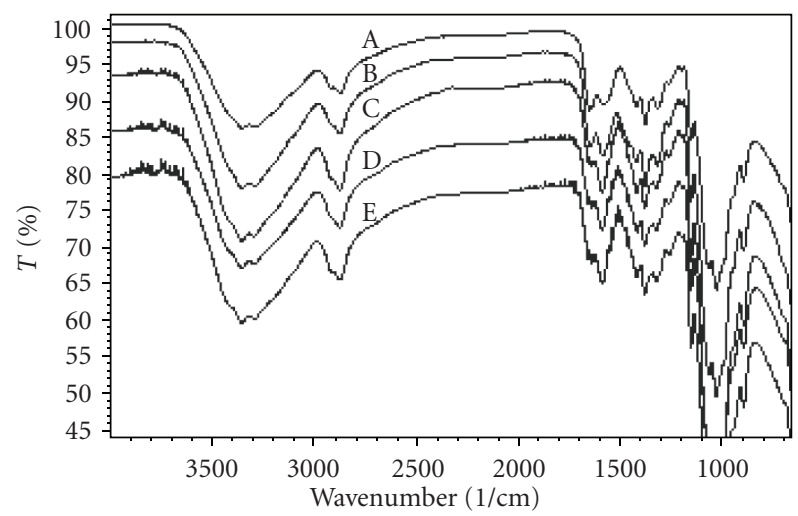

Figure 3: FTIR spectra of CHT1 (A), CHT2 (B), CHT3(C), CHT4 (D) and CHT5 (E).

of the solution. Initially the curve shows segments with an overlap concentration $\left(C^{*}\right)$ at the point of inflection and then flattens as the storage time is increased, that is, the critical concentration shifts towards right. It is also observed that the drop in viscosity in first $24 \mathrm{hrs}$ is much faster than the latter and is more significant at higher concentration, that is, above $C^{*}$. The loss in viscosity may be attributed to the biodegradation of chitosan molecules and/or hydrolysis of polymer molecules. Initially the large molecules, especially at higher concentration, occupy large "hydrodynamic" volume, which leads to less mobility indicating higher viscosity. The hydrolytic degradation of the polymer leads to the production of smaller molecular entities which in turn causes a drop in hydrodynamic volume of the polymer molecules resulting in higher molecular mobility and as a result reduces the viscosity of the solution $[19,27,28]$.

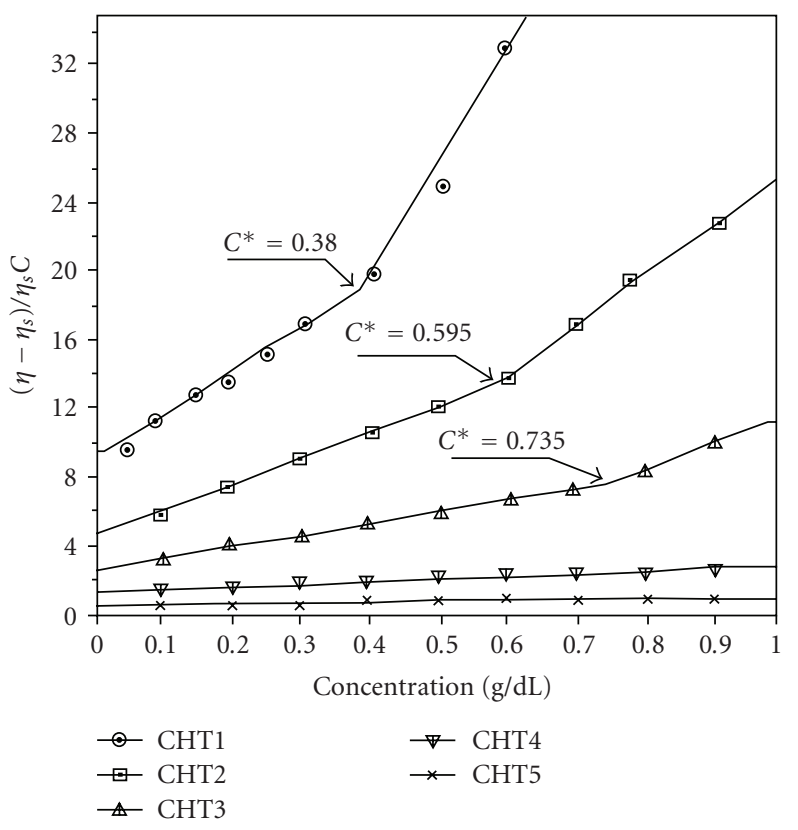

FIGURE 4: Intrinsic viscosities of different grades of chitosan.

3.3. Effect of Electrolyte on Viscosity of Chitosan. Chitosan is known to be a polycationic material; its solution is believed to be influenced by the presence of electrolytes. Therefore, the effect of sodium acetate concentrations on two different grades of chitosan namely, CHT 1 and CHT 3 was studied.

It can be seen from Figure 6 that the viscosity is sharply reduced in presence of sodium acetate up to a concentration of about $2 \mathrm{~g} / \mathrm{dL}$. Further increase in concentration has not influenced the viscosity much. It is also observed that the 


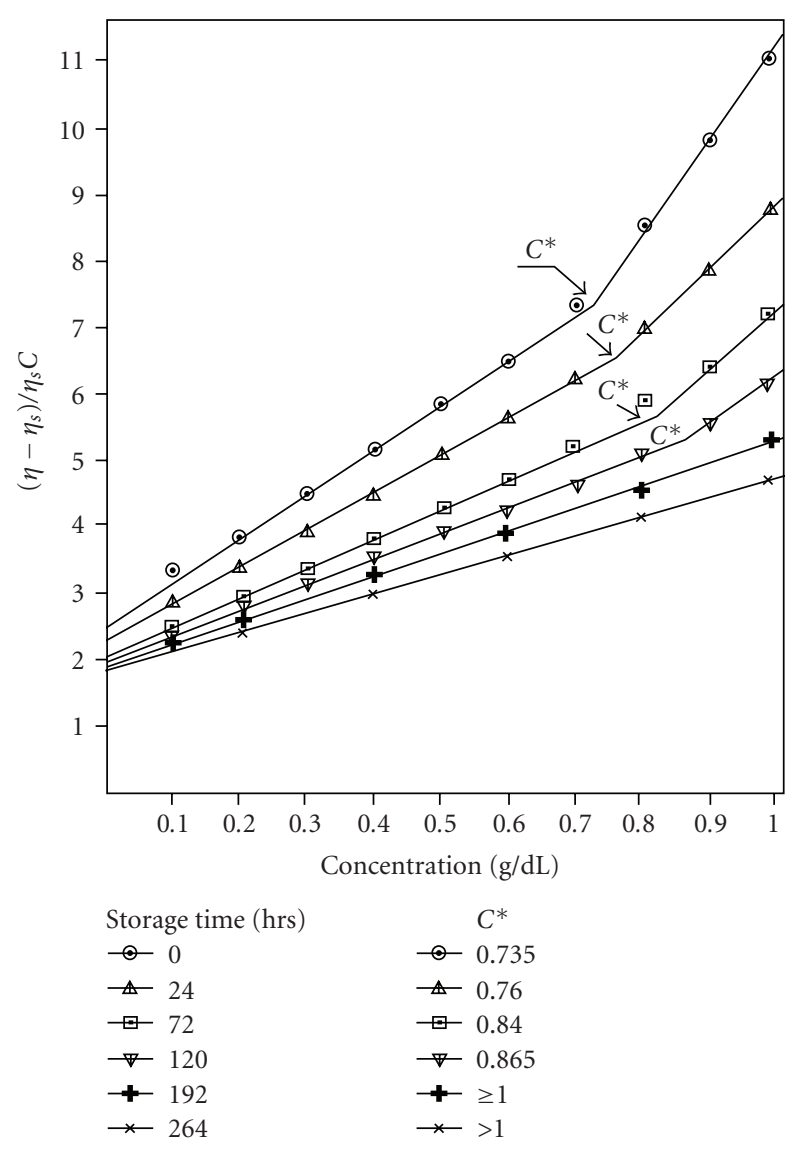

FIgURE 5: Viscosity of chitosan (CHT-3) solution as a function of storage time.

chitosan with higher molecular weight (CHT1) is more affected compared with its lower molecular counterpart (CHT2). The decrease in viscosity with increase in electrolyte concentration can be attributed to the shielding effect of counter ions [29]. Due to ion dipole forces, the acetate ions form a cascade of negative charge over each chitosan molecules establishing repulsive forces between them. This offers low resistance to the flow or mobility of the polymer molecules.

3.4. Effect of Particle Size on Viscosity of Chitosan. It is possible, for a given molecular size chitosan, to scale down the hydrodynamic volume to nanolevel by means of ionotropic gelation using suitable cross linking agent such as sodium tripolyphosphate (TPP), as illustrated in Figure 7. The particles are stabilized by electrostatic hindrance due to coulombic repulsion between particles of same ionic charges [30-32]. We prepared nanochitosan (CHT3N) of average size $394.8 \mathrm{~nm}$ from $0.25 \mathrm{gpl} \mathrm{CHT3}$. The particle size distribution and viscosity behaviour of this nanosolution are show in Figures 8 and 9 , respectively.

The particle size of chitosan is found to affect its viscosity behaviour significantly (Figure 9). The viscosity of nanochitosan colloid is dropped by about $30 \%$ with respect to normal chitosan solution at the same concentration level.

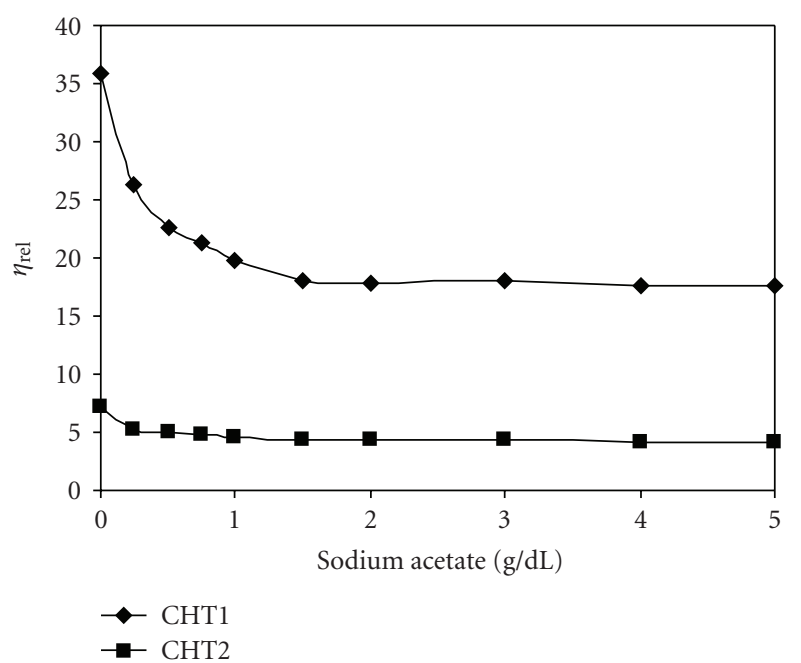

FIGURE 6: Relative viscosity of chitosan solution as a function of electrolyte concentration.
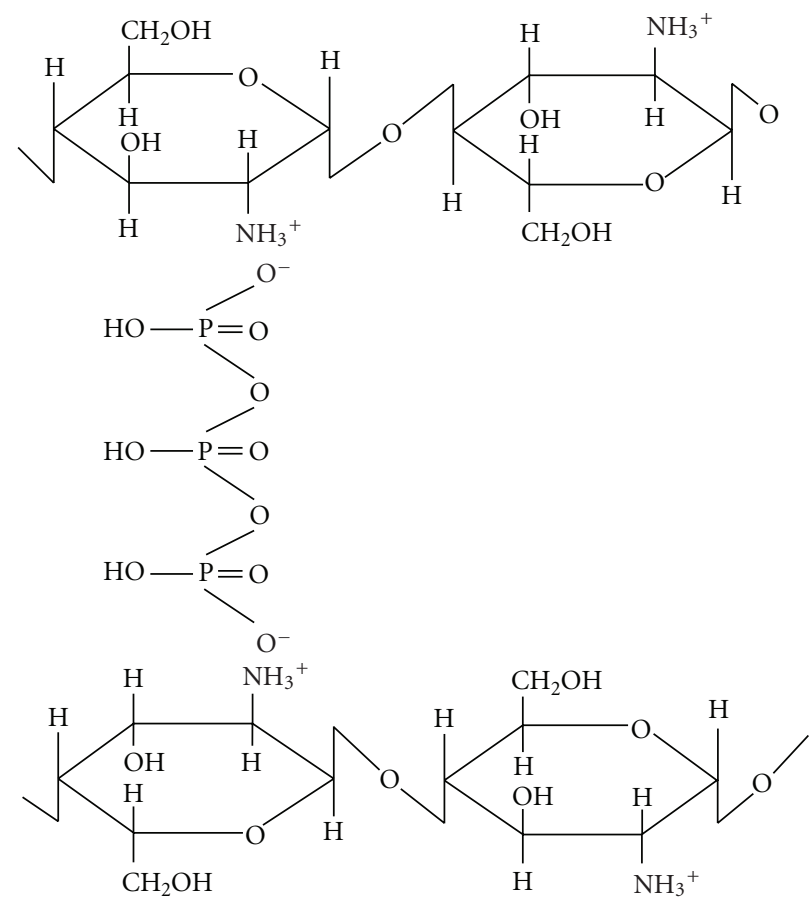

FIGURE 7: Ionotropic cross-linking of chitosan and TPP.

The reduction of size to nanolevel has also influenced the stability of chitosan solution. There is $10 \%$ drop in the viscosity of normal chitosan for a storage time of 24 hours, whereas nano colloids dropped by $17 \%$ for the same storage time. Thus reduction in size reduced the storage stability of the polymer. The reduction in viscosity of nano chitosan solution can be attributed to its lesser resistance towards flow due to its smaller size and enhanced degradation of the polymer in solution due to higher exposed surface area of the nanoparticles. 


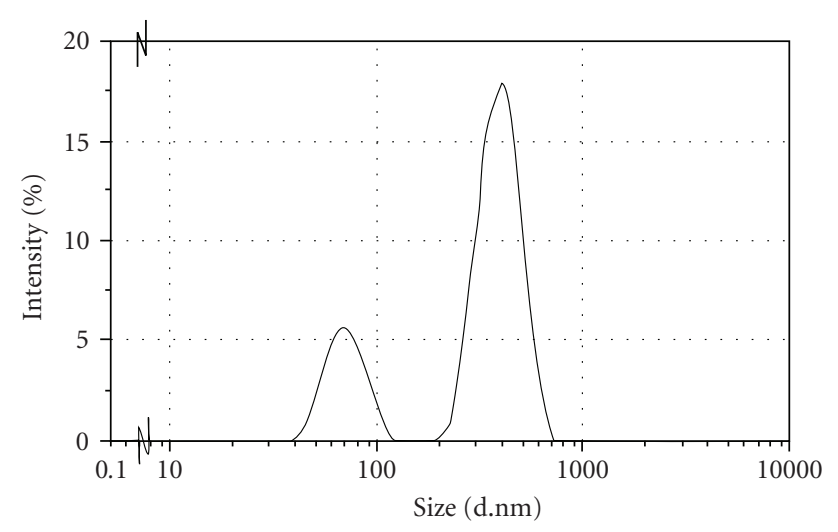

FIgURE 8: Size distribution of nanochitosan (CHT3N).

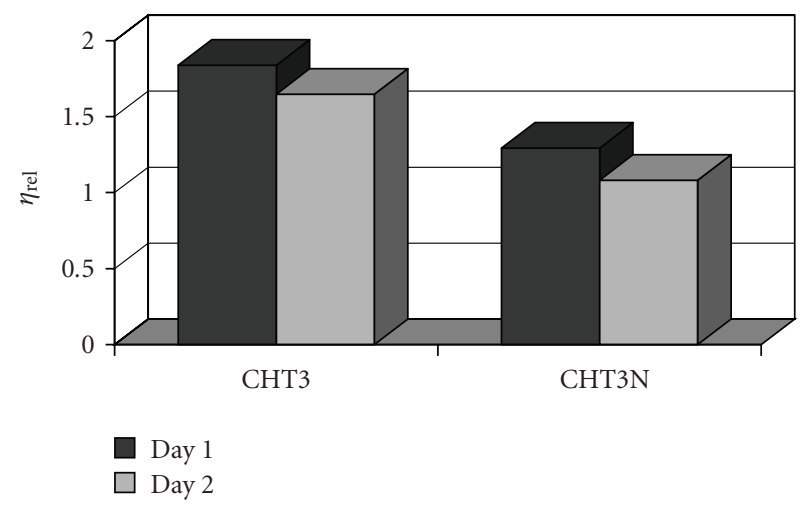

FIgURE 9: Relative viscosity of chitosan solution as a function of particle size.

3.5. Chelation Property of Chitosan. Attributing to the presence of large number of amino groups on chitosan backbone, this biopolymer can also be used in water processing engineering. Chitosan molecules are known to chelate anionic dyes in waste dye solution and flocculate them out.

It has also been found that the chitosan and its derivatives can remove phosphorus, heavy metals, and oils from water [33-37]. In order to study the chelation property of chitosan, the removal of calcium ions from water by CHT1 and CHT3 was determined and the results were compared with $\mathrm{Na}_{4}$ EDTA. Table 3 shows that chitosan can remove the calcium ions substantially though its sequestering power is found to be inferior to EDTA. Further, it is observed that maximum removal occurred in the first 2 hours. Higher molecular weight sample, CHT1 shows better chelation power compared with its lower molecular weight counterpart, CHT3. The former one also shows a continuation in chelation effect even after $96 \mathrm{hrs}$ without reaching the equilibrium, whereas the rest of the samples reached equilibrium within $24 \mathrm{hrs}$. A substantial higher chelation of $\mathrm{Na}_{4}$ EDTA may be attributed to the combined effect of both carboxylate and amino groups present in its molecule and possibly higher proximity of each molecule due to its relatively small size. The larger molecular size of chitosan provides less surface area and the less amino groups to form the coordinate linkage with calcium ions. The prolonged chelation effect of CHT1 is found probably due to gradual disentanglement of the polymer molecules with respect to time.

\section{Conclusions}

The viscosity of chitosan is influenced by its molecular weight. Curves of reduced viscosity verses concentration for higher molecular weight chitosan show inflection point, that is, a critical or overlap concentration $\left(C^{*}\right)$. The overlap concentration $\left(C^{*}\right)$ is a measure of molecular size and conformation of a polymer.

The higher the molecular weight and the more rigid is the conformation and lower is the value of $C^{*}$. The initial molecular weight and the concentration of chitosan are found to influence the stability of its solution. The drop in viscosity in first $24 \mathrm{hrs}$ is found to be very fast, and the critical/overlap concentration $\left(C^{*}\right)$ point shifts towards right when the storage time is prolonged.

In presence of electrolyte, the viscosity of chitosan solution is reduced, which is found to be more pronounced for high molecular weight chitosan solution.

By reducing the particle size to nanolevel, the viscosity of chitosan solution is lowered significantly, but the storage stability was affected adversely. Use of freshly prepared nanochitosan solution prior to applications may be the remedy.

Chitosan exhibits chelation property. This property is useful in removing metal and dye ions from water.

\section{References}

[1] S. Hirano, ULLMANN's Encyclopedia of Industrial Chemistry, vol. 7, Wiley-VCH, Weinheim, Germany, 6th edition, 2003.

[2] R. A. A. Muzzarelli, "Chitin chemistry," in The Polymeric Materials Encyclopedia, J. C. Salamone, Ed., pp. 312-314, CRC Press, Boca Raton Fla, USA, 1996.

[3] K. V. Harish Prashanth and R. N. Tharanathan, "Chitin/chitosan: modifications and their unlimited application potential-an overview," Trends in Food Science and Technology, vol. 18, no. 3, pp. 117-131, 2007.

[4] P. K. Dutta, J. Duta, and V. S. Tripathi, "Chitin and Chitosan: chemistry, properties and applications," Journal of Scientific \& Industrial Research, vol. 63, no. 1, pp. 20-31, 2004.

[5] Y.-S. Chung, K.-K. Lee, and J.-W. Kim, "Durable press and antimicrobial finishing of cotton fabrics with a citric acid and chitosan treatment," Textile Research Journal, vol. 68, no. 10, pp. 772-775, 1998.

[6] W. B. Achwal, "Chitosan and its derivatives for textile finishing," Colourage, vol. 50, no. 8, pp. 51-76, 2003.

[7] A. J. Rigby, S. C. Anand, and A. R. Horrocks, "Textile materials for medical and healthcare applications," Journal of the Textile Institute, vol. 88, no. 1, pp. 83-93, 1997.

[8] D. P. Chattopadhyay and M. S. Inamdar, "Studies on the properties of chitosan treated cotton fabric," Asian Dyer, vol. 6, no. 5, pp. 47-53, 2009.

[9] S. A. Bahmani, G. C. East, and I. Holme, "The application of chitosan in pigment printing," Journal of the Society of Dyers and Colourists, vol. 116, no. 3, pp. 94-99, 2000.

[10] P. Erra, R. Molina, D. Jocic, M. R. Julia, A. Cuesta, and J. M. D. Tascon, "Shrinkage properties of wool treated with 
low temperature plasma and chitosan biopolymer," Textile Research Journal, vol. 69, no. 11, pp. 811-815, 1999.

[11] D. Knittel and E. Schollmeyer, "Chitosan and its derivatives for textile finishing," Melliand Textilberichte, vol. 83, no. 1-2, pp. E15-E16, 2002.

[12] Z. Zhang, L. Chen, J. Ji, Y. Huang, and D. Chen, "Antibacterial properties of cotton fabrics treated with Chitosan," Textile Research Journal, vol. 73, no. 12, pp. 1103-1106, 2003.

[13] I. Holme, "Antimicrobials impart durable freshness," International Dyer, vol. 187, no. 12, pp. 9-11, 2002.

[14] M. A. Torres, A. M. Beppu, C. C. Santana, and E. J. Arruda, "Viscoelastic properties of chitosan solutions and gels," Brazilian Journal of Food Technology, vol. 9, no. 2, pp. 101-108, 2006.

[15] R. Shepherd, S. Reader, and A. Falshaw, "Chitosan functional properties,” Glycoconjugate Journal, vol. 14, no. 4, pp. 535-542, 1997.

[16] C. L. Velásquez, J. S. Albornoz, and E. M. Barrios, "Viscometric stidies of chitosan nitrate and chitosan chlorhydrate in acid free $\mathrm{NaCl}$ aq solution," e-Polymers, no.014, 2008.

[17] M. Sano, O. Hosoya, S. Taoka et al., "Relationship between solubility of chitosan in alcoholic solution and its gelation," Chemical and Pharmaceutical Bulletin, vol. 47, no. 7, pp. 10441046, 1999.

[18] S. K. Tiwari and M. M. Gharia, "Characterization of chitosan pastes and their application in textile printing," AATCC Review, vol. 3, no. 4, pp. 17-19, 2003.

[19] V. R. Gowariker, N. V. Viswanathan, and Y. Sreedhar, "Polymer solutions," in Polymer Science, pp. 332-362, New Age International, New Delhi, India, 1986.

[20] A. Hebeish, A. Waly, and A. Aou-Okeil, "The effect of molecular weight of chitosan on cotton fabric treated with citric acid and its impact on dyeing with some acid dyes," Journal of the Textile Association, vol. 65, no. 5, pp. 219-227, 2005.

[21] H. S. Seong, J. P. Kim, and S. W. Ko, "Preparing chitooligosaccharides as antimicrobial agents for cotton," Textile Research Journal, vol. 69, no. 7, pp. 483-488, 1999.

[22] F. Lee, W. K. Lee, M. Y. Maskat et al., "Partial depolymerization of chitosan with the aid of bromelain," Pakistan Journal of Biological Sciences, vol. 8, no. 1, pp. 73-77, 2005.

[23] F. Tian, Y. Liu, K. Hu, and B. Zhao, "The depolymerization mechanism of chitosan by hydrogen peroxide," Journal of Materials Science, vol. 38, no. 23, pp. 4709-4712, 2003.

[24] S. Trzciński, "Combined degradation of chitosans," Polish Chitin Society, Monograph, vol. 11, pp. 103-111, 2006.

[25] D. J. Shaw, "Rheology," in Introduction to Colloid and Surface Chemistry, pp. 244-260, Butterworth-Heinemann, Oxford, UK, 4th edition, 1992.

[26] J. Z. Knaul, V. T. Bui, K. A. M. Creber, and M. R. Kasaai, "Characterization of deacetylated chitosan and chitosan molecular weight review," Canadian Journal of Chemistry, vol. 76, no. 11, pp. 1699-1706, 1998.

[27] A. Tager, "Rheological properties of polymers in viscofluid state," in Physical Chemistry of Polymers, pp. 241-272, MIR Publishers, Moscow, Russia, 1972.

[28] J. K. Hwang and H. H. Shin, "Rheological properties of chitosan solutions," Korea-Australia Rheology Journal, vol. 12, no. 3-4, pp. 175-179, 2000.

[29] M. Terbojevich and R. A. A. Muzzarelli, "Chitosan," in Handbook of Hydrocolloids, G. O. Phillips and P. A. Williams, Eds., pp. 367-378, Woodhead Publishing, Cambridge, UK, 2000.
[30] R. D. Bhumkar and V. B. Pokharkar, "Studies on effect of pH on cross-linking of Chitosan with sodium tripolyphosphate: a technical note," AAPS PharmSciTech, vol. 7, no. 2, pp. E1-E6, 2006.

[31] Y. Wu, W. Yang, C. Wang, J. Hu, and S. Fu, "Chitosan nanoparticles as a novel delivery system for ammonium glycyrrhizinate," International Journal of Pharmaceutics, vol. 295, no. 1-2, pp. 235-245, 2005.

[32] T. López-León, E. L. S. Carvalho, B. Seijo, J. L. Ortega-Vinuesa, and D. Bastos-González, "Physicochemical characterization of chitosan nanoparticles: electrokinetic and stability behavior," Journal of Colloid and Interface Science, vol. 283, no. 2, pp. 344351, 2005.

[33] S. M. Nomanbhay and K. Palanisamy, "Removal of heavy metal from industrial wastewater using chitosan coated oil palm shell charcoal," Electronic Journal of Biotechnology, vol. 8, no. 1, pp. 43-53, 2005.

[34] B. Smith, T. Koonce, and S. Hudson, "Decolorizing dye wastewater using chitosan," American Dyestuff Reporter, vol. 82, no. 10, pp. 18-66, 1993.

[35] J. A. Laszlo, "Removing acid dyes from textile wastewater using biomass for decolorization," American Dyestuff Reporter, vol. 83, no. 8, pp. 17-21, 1994.

[36] P. K. Dutta, K. Durga Bhavani, and N. Sharma, "Adsorption for dyehouse effluent by low cost adsorbent (Chitosan)," Asian Textile Journal, vol. 10, no. 1, pp. 57-63, 2001.

[37] H. Ge and S. Huang, "Microwave preparation and adsorption properties of EDTA-modified cross-linked chitosan," Journal of Applied Polymer Science, vol. 115, no. 1, pp. 514-519, 2010. 

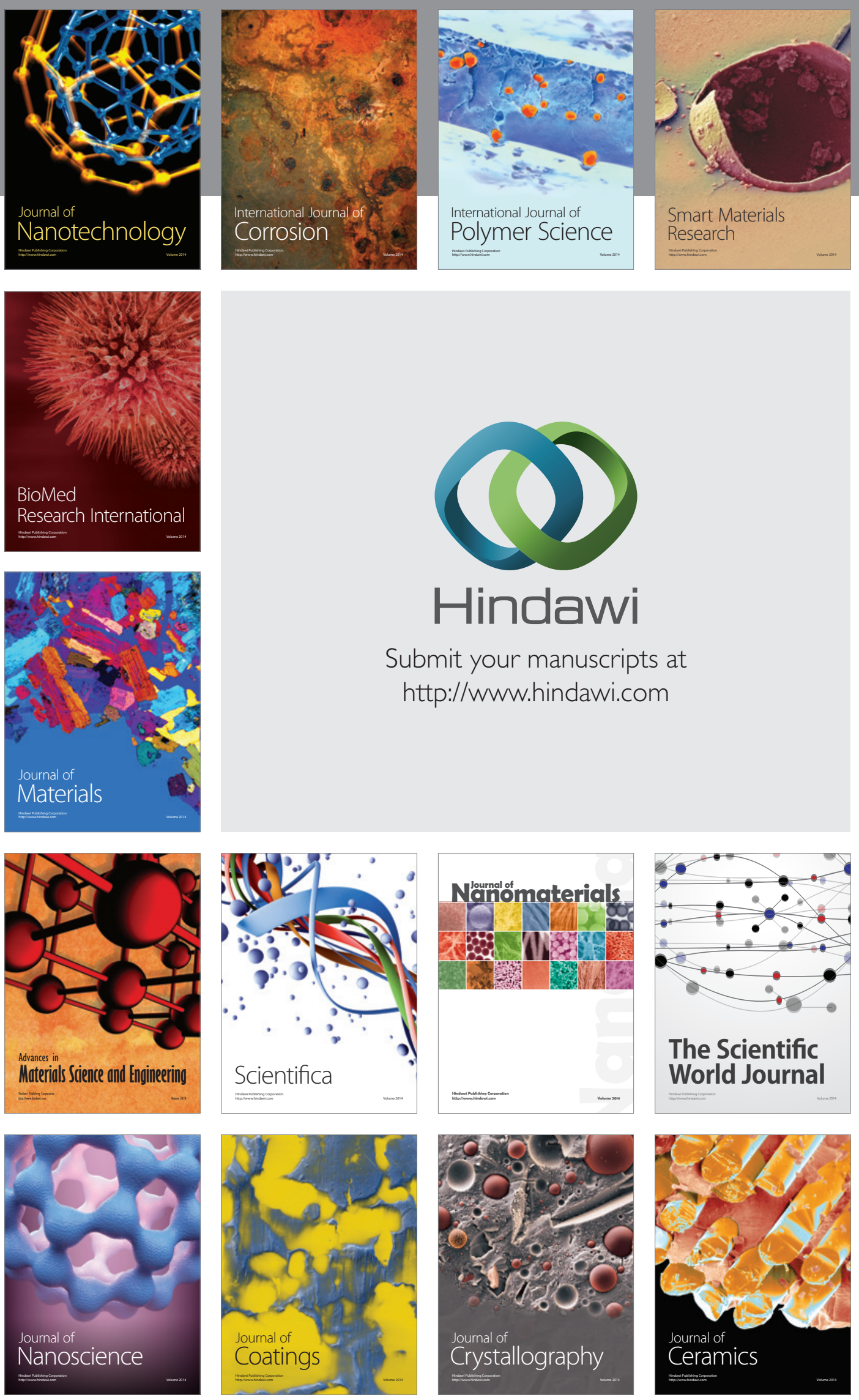

The Scientific World Journal

Submit your manuscripts at

http://www.hindawi.com

\section{World Journal}

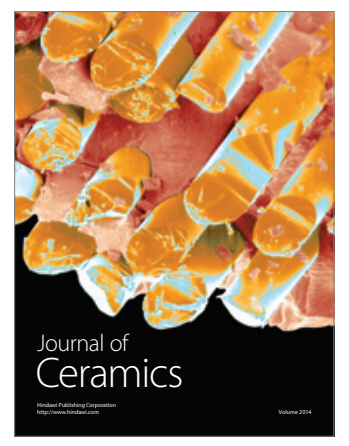

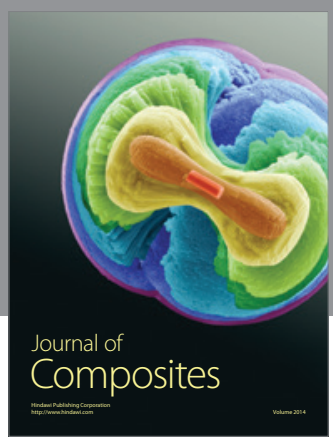
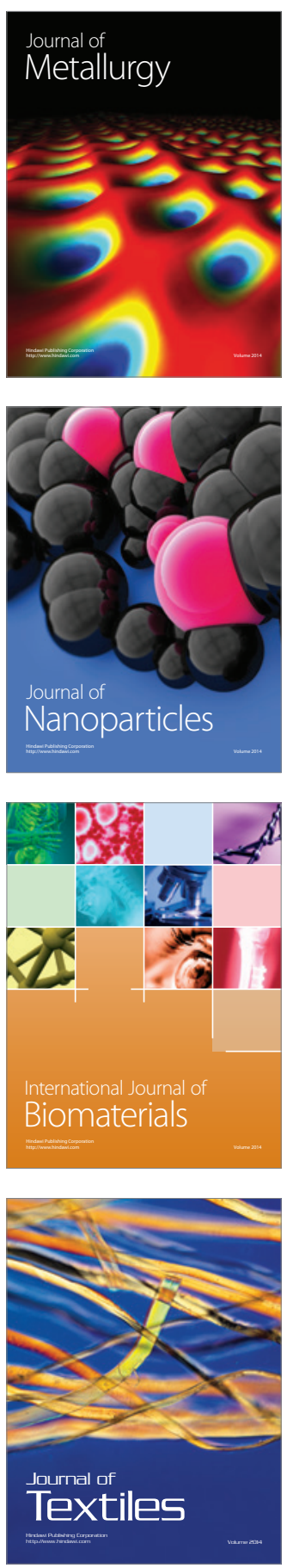